Article

\title{
Activation of microbiota sensor- free fatty acid receptor 2 signaling ameliorates amyloid- $\beta$ induced neurotoxicity by modulating proteolysis/senescence axis
}

Atefeh Razazan ${ }^{\sharp}$, Prashantha Karunakar2, Sidharth P Mishra1 ${ }^{1}$, Shailesh Sharma ${ }^{3}$, Shalini Jain ${ }^{4}$, Hariom Yadav ${ }^{1,5^{*}}$

1 Department of Internal Medicine- Molecular Medicine, Wake Forest School of Medicine, Winston-Salem, NC, USA

2 PES University, Bangalore, Karnataka, India

3 National Institute of Animal Biotechnology, Hyderabad, NC, India

4 Department of Internal Medicine- Endocrinology, Wake Forest School of Medicine, Winston-Salem, NC, USA

5 Department of Microbiology and Immunology, Wake Forest School of Medicine, Winston-Salem, NC, USA

* Current affiliation: West Virginia University, Morgantown, WV, USA

* Correspondence: hyadav@wakehealth.edu; Tel.: +01-336-716-5049 (L)

\begin{abstract}
Gut microbiota and its metabolites like short chain fatty acids (SCFAs) are linked with pathology of Alzheimer's disease (AD) - a debilitating public health problem in older adults. However, strategies to beneficially modulate gut microbiota and its sensing signaling pathways remain largely unknown. Here, we screened, validated and established the agonists of free fatty acid receptor 2 (FFAR2) signaling, which senses beneficial signals from SCFAs produced by microbiota in the gut. We demonstrated that inhibition of FFAR2 signaling increases amyloid-beta (A $\beta$ ) stimulated neuronal toxicity. Thus, we screened FFAR2 agonists, using in-silico library of more than 144,000 natural compounds, and 15 compounds were selected based on binding with FFAR2 agonist sites. Further, cell culture toxicity and FFAR2 stimulatory experiments demonstrated that Fenchol (a natural compound commonly present in basil) was potent FFAR2 agonist in neuronal cells. Interestingly, we also demonstrated that Fenchol protects $A \beta$-stimulated neurodegeneration in FFAR2 dependent manner. In addition, Fenchol reduced AD like phenotypes such as $A \beta-$ accumulation and, learning and memory behaviors in Caenorhabditis (C.) elegans. Fenchol increased $\mathrm{A} \beta$-clearance by increasing proteasome/lysosome activity and reduced senescence in neuronal cells. These results demonstrated that the inhibition of FFAR2 signaling promotes $A \beta$-induced neurodegeneration, while activating it by Fenchol as a natural agonist reverse it by promoting $\mathrm{A} \beta$ clearance and reducing cellular senescence; thus stimulation of FFAR2 signaling can be a therapeutic approach to prevent/ treat AD.
\end{abstract}

Keywords: Free fatty acid receptor 2, microbiota, metabolite, sensor, G-coupled protein receptor, signaling, Alzheimer's disease, senescence, C. elegans

\section{Introduction}

Alzheimer's disease (AD) is the most common neurodegenerative disorder in older adults. Its prevalence is increasing and no successful prevention and treatments are available. AD is commonly characterized with high accumulation of amyloid-beta $(A \beta)$, formation of intracellular neurofibrillary tangles and neuronal death, with loss in memory and learning behaviors [1,2]. Although mechanisms by which accumulation of $\mathrm{A} \beta$ (an important hallmark of $\mathrm{AD}$ ) increases in brain are not known, however, their accumulation increases neurotoxicity/neuronal death (neurodegeneration) and impaired learning and memory behaviors [2-4]. Increased A $\beta$ accumulation are associated with either 
higher production or reduced clearance [5]. Reduced clearance is major hallmark in this process, which is linked due to reduced proteolytic lysosomal activity in brain [6,7]. Increased $A \beta$ accumulation is known to stimulate senescence in neuronal and microglial cells, that initiate a cascade of detrimental events including neuroinflammation, that further deteriorate brain health and functions like memory and learning behaviors [8]. However, strategies to reduce burden of $A \beta$ and $\mathrm{AD}$ pathology are lacking, and one of the reason is because we do not fully understand the pathology of $A \beta$ accumulation and their contribution in $A D$ pathology, as well as precise mechanisms to target to mitigate their accumulation.

Multiple emerging evidence including our recent studies demonstrated that the gut microbiota signature is significantly different in the patients with $\mathrm{AD}$ and/or with mild impairment (MCI- an early stage of $\mathrm{AD}$ ) $[9,10]$. Indeed, few studies demonstrated that restoring abnormalities of gut microbiota to normal using fecal microbiota transplantation decreases the progression of AD [11], suggesting a causal role instead of mere link. Although, mechanisms by which gut microbiota influence brain health is under intensive research, however, it is known that the metabolites produced by gut microbiota can efficiently impact brain health [12-15]. Short chain fatty acids (SCFAs; such as acetate, propionate and butyrate) are the major metabolites produced by healthy gut microbiota and SCFAs exhibit beneficial effects on host including reducing AD pathology in several animal models [16-18]. Indeed, the abundance of SCFAs is commonly reduced in the gut of patients with AD and $\mathrm{MCI}$, while healthy dietary habits like ketogenic diet increases their production $[9,19]$. However, the clinical evidence of SCFAs benefit on AD remain unknown. In addition, although, SCFAs are available for human consumption as a dietary supplement, however, SCFAs therapies are limited due to the fact that- (i) these formulations are in salt form, and their efficacy requirements of 4-5 grams/day are restrictive to be used in AD patients- because most of them are older and on high risk of salt related ailments; and (ii) the SCFAs are digestible, thus absorbs in upper gut and becomes energy supplier instead of biofunctional, while produced in lower gut. Thus, developing alternatives for stimulating SCFAs effect on brain can be beneficial to ameliorate AD pathology.

The well-known mechanisms by which SCFAs act on target cells including neurons are primarily of two types- (i) either they activate free fatty acid receptor 2 and 3 (FFAR2/3)- an inhibitory G (Gi)-couple protein receptor signaling; and/or (ii) they enter into target cells and change the intracellular metabolic flux and/or inhibit histone deacetylase activity, causing wide-spectrum gene expression changes [16]. However, the mechanisms of these signaling in AD progression remain largely unknown. Here, we focus on FFAR2 signaling, because we and others have demonstrated that FFAR2 is widely expressed in gut and brain, including in neuronal cells, and its inhibition cause higher $A \beta$-induced neurotoxicity. In this study, we screened, validated and established evidence for new FFAR2 agonists of natural origin, using more than 144,000 natural compounds libraries. We discovered that Fenchol (a terpenoid commonly present in Basil) found to be most effective FFAR2 agonist and reduced $A \beta$-induce neurotoxicity in neuronal cells and animal models like $C$. elegans. Mechanistically, Fenchol increases A $\beta$ clearance by increasing proteasome and lysosome activity, and reduces senescence in neuronal cells.

\section{Materials and Methods}

\subsection{Measurement of the expression of FFAR2 in SK-N-SH neuronal cells}

We measured FFAR2 expression in human neuronal cells SK-N-SH and compared with human kidney HEK293 cells and mouse enteroendocrine Glutag cells as positive controls. Cells were cultured in DMEM medium (with 10\% FBS and 1\% v/v penicillin and streptomycin) at $37^{\circ} \mathrm{C}$ till 70 $80 \%$ confluency and total RNA was isolated using RNAeasy kit (Qiagen). The cDNA was synthesized using High-Capacity cDNA Reverse Transcription kit (Applied Biosystems) and expression of FFAR2 mRNA was quantified using real-time PCR assay. $18 \mathrm{~S}$ has been used as internal control. The fold change in gene expression was calculated using delta-delta Ct values, following our well-established and published methods [20-24]. 


\subsection{Determine the impact of FFAR2 inhibition on A $\beta$-induced neurotoxicity using MTT assay}

To determine the effects of FFAR2 inhibition on A $\beta$-induced neuronal cell-toxicity, we performed MTT viability assay. SK-N-SH cells $\left(1 \times 10^{3}\right)$ per well were plated in 96-well plate containing $10 \% \mathrm{FBS}$ and $1 \%$ antibiotics (penicillin and streptomycin) supplemented DMEM and grown at $37^{\circ} \mathrm{C}$ for $24 \mathrm{~h}$. After removing media and washing with PBS, cells were treated with $10 \mu \mathrm{M}$ FFAR2 inhibitor (CATPB, TOCRIS) dissolved in 1\% FBS supplemented DMEM, 2 hrs before treating cells with $A \beta 25-32\left(25 \mu \mathrm{M}\right.$, Sigma) peptide. After incubation for $4 \mathrm{~h}$ at $37^{\circ} \mathrm{C}$, the media was replaced with $100 \mu \mathrm{L}$ of fresh media. The MTT reagent $(5 \mathrm{mg} / \mathrm{ml}$ in phosphate buffer [pH 7.2]) was dispensed $(10 \mu \mathrm{L})$ in each well and incubated for 4 hrs. Following DMSO $(50 \mu \mathrm{L})$ was added in each well and mixed thoroughly to dissolve formazan crystals. The absorbance was measured at $570 \mathrm{~nm}$.

\subsection{In-silico screening of natural FFAR2 agonists and identification of these molecules}

Protein primary sequence of human and mouse FFAR2 were retrieved from the Uniprot Database in FASTA format with the Uniprot IDs O15552 and Q8VCK6, respectively. The structures of 144,356 natural compounds were obtained from ZINC database (Supplementary Table S1). Through in-silico virtual screening of these compounds based on binding to human FFAR2 using DOCK6 software, we shortlisted top 15 compounds (Supplementary Table S2). Further, the tertiary structure of both mouse FFAR2 and human FFAR2 were predicted using the threading based online server I-TASSER [25-27], refined using ModRefiner [28] and validated using the Ramachandran plot from RAMPAGE server [29]. The natural agonists, both acetate and butyrate along with the shortlisted compounds were blindly docked with both mouse FFAR2 and human FFAR2 using AutoDock Vina to find the compounds that shows agonist property [30]. AutoGrid program was used to fix the grid box around the proteins. The grid box for the human FFAR2 binding site was set to the XYZ coordinates of $68.028,66.787$ and 54.804 respectively. The box dimensions were $28.089,28.562$ and 25.349 along the XYZ axis respectively. For the mouse FFAR2, the grid box binding site was set to XYZ coordinates of $63.359,66.205$ and 46.924 and the box dimensions were $40.229,41.757$ and 36.283 respectively. The nine conformations along with the Vina score was used to screen the agonists. The interaction of the ligands were analyzed using the 2D interaction diagrams generated by using LigPlot+ [31] and 3D interaction images using PyMOL2.3 (The PyMOL Molecular Graphics System, Version 2.0 Schrödinger, LLC).

\subsection{Measurements of the ERK1/2 signaling using Western blotting}

To determine the FFAR2 signaling activating property of selected compounds, we treated SK$\mathrm{N}-\mathrm{SH}$ cells with $10 \mu \mathrm{M}$ of shortlisted compounds and cells were harvested after $30 \mathrm{~min}$ at $37^{\circ} \mathrm{C}$. The cells were washed with cold PBS (1X) and total protein was extracted from cells by homogenizing them in lysis buffer $(10 \mathrm{mmol} / \mathrm{L}$ Tris $\mathrm{pH} 7.6,150 \mathrm{mmol} / \mathrm{L} \mathrm{NaCl}, 10 \mathrm{mmol} / \mathrm{L}$ Sodium orthovanadate, $10 \mathrm{mmol} / \mathrm{L}$ Sodium Pyrophosphate, $100 \mathrm{mmol} / \mathrm{L}$ Sodium fluoride, $1 \mathrm{mmol} / \mathrm{L}$ EDTA, $1 \mathrm{mmol} / \mathrm{L}$ EGTA, $1 \%$ Triton X-100, 0.5\% NP-40 and cocktail of protease inhibitors (Roche)). After centrifuging at $18000 \mathrm{~g}$ for $10 \mathrm{~min}$ at $4^{\circ} \mathrm{C}$, total protein concentration was measured in the collected supernatant using BCA protein assay kit. Equal amount of total protein $(45 \mu \mathrm{g})$ from each sample was loaded in corresponding wells by mixing them with loading buffer. Proteins were resolved on $4 \%$ stacking and $10 \%$ separating SDS-PAGE gel at $100 \mathrm{~V}$ current. Following, proteins were transferred on a 0.22 $\mu \mathrm{m}$ PVDF membrane and blots were developed with anti-ERK1/2, anti-phosphoERK1/2, ubiquitin (P4D1) and A $\beta$ (6E10) primary antibodies following with their secondary antibodies and bands were visualized using the Pierce ${ }^{\mathrm{TM}}$ Fast Western Blot Kit, ECL Substrate acquired using Syngene Pxi. Band densities were also quantified using NIH ImageJ software. $\beta$-actin has been used as an internal control. 
FFAR2 is a Gi/o signaling, which reduces cytosolic cAMP, thus to determine the FFAR2 signaling activating potential, we also measured cAMP using cAMP-GloTM Assay. In brief, SK-NSH cells ( $1 \times 10^{3}$ cells) per well were plated in white 96-well plate. After an overnight incubation, the cells were treated with $10 \mu \mathrm{M}$ compounds, and $10 \mu \mathrm{M}$ forskolin (a positive control) dissolved in induction buffer. The cAMP levels were detected using detection solution using Luminescence Microplate Reader.

\subsection{Measurement of intracellular calcium}

FFAR2 signaling increases intracellular calcium, thus to further determine the activation potential of selected compounds in neuronal cells. The SK-N-SH $\left(1.25 \times 10^{3}\right)$ cells were plated into dark bottom 96- well plate, then treated with $10 \mu \mathrm{m}$ compound(s) and $10 \mathrm{mM}$ acetate (a SCFA control). Also, $0.01 \mathrm{M}$ EDTA (Sigma) and $114 \mathrm{~nm}$ carbachol were used as negative and positive controls. After an overnight incubation, the cells were treated with $2 x$ fluo- 4 DirectTM calcium reagent along with $250 \mathrm{mM}$ probenecid and further incubated at $37^{\circ} \mathrm{C}$ for 30 minutes. The fluorescence was recorded with excitation at $494 \mathrm{~nm}$ and emission at $516 \mathrm{~nm}$ using microplate reader.

\subsection{C. elegans strains, their culture and maintenance}

We have used C. elegans as a model for determining the impact of selected compounds on AD like phenotype. The wild-type (N2) and CL2122 (dvls15 [9pPD30.38) unc-54(vector)+(pCL26)mtl2::GFP]) were propagated at $20^{\circ} \mathrm{C}$. AD transgenic worms model CL2006 (dvls [ pCL12(unc54/human abeta peptide 1-42 minigene) + pRF4), CL4176 (dvls27[myo-3p::A-Beta (1-42)::let-851 3'UTR) + rol-6 (su1006] and CL2355 (dvls 50 [ pCL45 9snb-1::Abeta 1-42::3' UTR 9log) + mtl-2::GFP]) were maintained at $16^{\circ} \mathrm{C}$. All strains were cultured on solid nematode growth medium (NGM) $3 \mathrm{~g} / \mathrm{L} \mathrm{NaCl}, 2.5 \mathrm{~g} / \mathrm{L}$ peptone, $5 \mathrm{mg} / \mathrm{L}$ cholesterol, $1 \mathrm{mM} \mathrm{CaCl}$, $1 \mathrm{mM} \mathrm{MgSO} 4,25 \mathrm{mM} \mathrm{KH} 2 \mathrm{PO} 4$, and $17 \mathrm{~g} / \mathrm{L}$ agar) and seeded with $50 \mu \mathrm{OP} 50$ (Escherichia coli strain) as a food supply. The worms were allowed to lay eggs for an overnight to prepare age-synchronized nematodes [32]. Then, isolated the adult worms from the synchronized eggs (day 1) and the synchronized eggs were cultured to the NGM plate or fresh S-Complete plus 5\% OP50, containing $10 \mu \mathrm{M}$ Fenchol (Sigma) in either $20^{\circ} \mathrm{C}$ (N2 and CL2122) or a $16^{\circ} \mathrm{C}$ (CL2006, CL4176 and CL2355). All of strains used in this study were acquired from the University of Minnesota- Caenorhabditis Genetics Center, which is funded by NIH Office of Research Infrastructure Programs (P40 OD010440).

\subsection{Lifespan and paralysis assays in C. elegans}

To determine the impact of selected compounds on age-related decline in brain health and lifespan, we measured the life-span of the worms. The CL4176 and CL2006 worms were grown at $16^{\circ} \mathrm{C}$ and $\mathrm{N} 2$ at $20^{\circ} \mathrm{C}$. The L1 worms were cultured on S-complete plus OP50 and antibiotics in 96-plates (about 15-10 animals per well). The worms were treated with $10 \mu \mathrm{m}$ Fenchol and 0.1\% DMSO was used as control. The $0.6 \mathrm{mM}$ Fluorodeoxyuridine (FUDR; 30 $\mu \mathrm{l}$ ) was added to each well after 3 days; and animals were monitored on every second day movements.

For paralysis assay, synchronized eggs of CL4176 were kept at $16^{\circ} \mathrm{C}$ for $48 \mathrm{~h}$, after that worms were transferred on the NGM plates and treated with $10 \mu \mathrm{m}$ Fenchol and DMSO as a control group at $25^{\circ} \mathrm{C}$ for $20 \mathrm{~h}$. The counting was performed at $2 \mathrm{hrs}$ interval typically after $14 \mathrm{~h}$ at $23^{\circ} \mathrm{C}$ until the last worm became paralyzed. If worms that did not move or only moved their head but not the body in response when gently touched with a platinum loop were scored as paralyzed. Each $C$. elegans experiment was conducted with at least 100 worms.

\subsection{Fluorescent thioflavin-S- staining of beta amyloids}


For detecting amyloid plaques in the transgenic CL2006, age-synchronized worms fed with or without Fenchol were incubated at 5 days of age; and were fixed in $4 \%$ paraformaldehydephosphate-buffered saline (PBS) after $24 \mathrm{hrs}$. The body of worms was permeabilized in $1 \%$ Triton X100, $125 \mathrm{mM}$ Tris, $\mathrm{pH} 7.4,5 \%$ fresh $\beta$-mercaptoethanol; and after washing 3-times with $1 \%$ PBSTween, and stained with $0.125 \%$ thioflavin $S$ in $50 \%$ ethanol for $2 \mathrm{~min}$. The samples were washed 3 times again with ethanol in dark room, mounted on slides for microscopy and after overnight samples observed with a Zeiss 710 confocal microscope equipped with a digital camera. A total of 20 worms were counted for each group. The data were the representatives of at least 3 independent experiments.

\subsection{Measurement of chemotaxis activity}

The CL2122 and Cl 2355 were treated with $10 \mu \mathrm{g} / \mathrm{ml}$ Fenchol and DMSO. They were cultured at $16^{\circ} \mathrm{C}$ for $36 \mathrm{~h}$, and then at $23^{\circ} \mathrm{C}$ for another $36 \mathrm{~h}$. Then the worms were washed twice with S-Basal media and transferred to an NGM plate without E. coli OP50. These plates are divided into four equal sectors; and $1 \mu \mathrm{l}$ of $0.1 \%$ benzaldehyde as a odorant along with $1 \mu \mathrm{l}$ of $1 \mathrm{M}$ sodium azide as an anesthetic to arrest the worms upon reaching a quadrant) were added to the original spot. On the opposite side of the attractant, $1 \mu \mathrm{l}$ drop of sodium azide and $1 \mu \mathrm{l}$ of control odorant $(100 \%$ ethanol) were added on NGM agar plate. Around 40-45 worms were placed to the center of each plate; and incubated at room temperature for $1 \mathrm{~h}$ and transferred to $4^{\circ} \mathrm{C}$ for overnight. Chemotaxis index (CI) was scored using formula as (number of worms at the attractant location - number of worms at the control location)/total number of worms on the plate.

\subsection{Measurement of proteasome activity}

The CL2006 strain and SK-N-SH cells were sonicated in a lysis buffer $(150 \mathrm{mM} \mathrm{NaCl}, 50 \mathrm{mM}$ HEPES, $2 \mathrm{mM}$ DTT, $20 \mathrm{mM} \mathrm{NaF}$ 5, mM EDTA, $2.5 \mathrm{mM}$ sodium pyrophosphate, $1 \mathrm{mM}$ sodium orthovanadate and $1 \mathrm{mM} \alpha$-glycerophosphoric acid) and then centrifuged at $15,000 \times \mathrm{g}$ at $4{ }^{\circ} \mathrm{C}$ for $15 \mathrm{~min}$. Total protein concentration was assayed by BCA kit and $20 \mu \mathrm{g}$ protein was mixed with 140 $\mu \mathrm{M}$ suc-leu-leu-val-tyr-7-amino-4-methylcou-marin (AMC) in a proteasome activity assay buffer (150 mM NaCl, 50 mM HEPES pH 7.4, $5 \mathrm{mM}$ ATP and 5 mM EDTA); and then fluorescence (excitation $380 \mathrm{~nm}$ and emission $460 \mathrm{~nm}$ ) was measured every 10 min over $3 \mathrm{~h}$ by a microplate reader. Assay was performed with and without of the proteasome inhibitor MG132 $(10 \mu \mathrm{M})$. Experiments were performed in triplicate.

\subsection{Impact of Fenchol treatment on lysosomal activity in the brain of APP/PS1 mice}

The impact of Fenchol on lysosomal activity of AD mice model- APP/PS1 was evaluated using five weeks older APP/PS1 mice and treated with Fenchol $(80 \mathrm{mg} / \mathrm{kg})$ by daily oral gavage for 3 months compared to non-treated controls. Brains were collected and immediately homogenized in proteasome lysis buffer and total protein was assayed by BCA kit. Measurement of proteasome activity was measured as described above. All the animal procedures and experiments were approved from institutional animal care and use committee of the Wake Forest School of Medicine.

\subsection{Measurements of lysosome activity}

The lysosomal activity in SK-N-SH cells were measured in $1 \times 10^{3}$ cells plated into 96- well plate and treated with $10 \mu \mathrm{m}$ fenchol, $25 \mu \mathrm{m}$ A $\beta 25-32,1 \%$ DMSO and $500 \mathrm{nM}$ bafilomycin. Bafilomycin used as an inhibitor of cellular autophagy/lysosomal activity. Lysosomal activity was assayed with Enz® lysozyme assay kit (E-22013, Molecular Probes) and the fluorescence was measured in a fluorescence microplate reader using excitation/emission of 494/518 nm. 
The effects of selected compounds on senescence in SK-N-SH cells were measured. Cells were treated with $10 \mu \mathrm{l} \mathrm{Fenchol} \mathrm{and} \mathrm{DMSO,} \mathrm{and} \mathrm{after} \mathrm{overnight} \mathrm{incubation} \mathrm{A} \beta 25-32(25 \mu \mathrm{M})$ peptide (Sigma) was added to cells for $4 \mathrm{~h}$. The Senescence $\beta$-Galactosidase Staining Kit (Cell Signaling, USA) was used for detection of expression of $\beta$-galactosidase activity, following the manufacturer's instructions.

\subsection{Statistical analyses.}

Student $t$-test and ANOVA have been used to determine the statistical significance, wherever was applicable. Values are presented as means and standard error means. Values with less than 0.05 values were considered statistically significant.

\section{Results}

\subsection{Inhibition of FFAR2 increases $A \beta$ induced neurotoxicity}

To determine the role of FFAR2 in neuronal function, first we confirmed and found that FFAR2 gene is abundantly expressed in human neuronal SK-N-SH cells similar to HEK293-used as a positive control (Fig. 1a). We also established that A $\beta$ - treatment significantly induces cell death in SK-N-SH cells compared to non-treated controls (Fig. 1b). Interestingly, we also observed that inhibition of FFAR2 using small compound inhibitor (CATPB) further exacerbated A $\beta$-induced cell death in these human neuronal cells, suggesting that FFAR2 inhibition increases detrimental effects of $A \beta$ on survival of neuronal cells. Thus, activation of FFAR2 signaling may be beneficial for protecting A $\beta$ induced neuronal cell death.
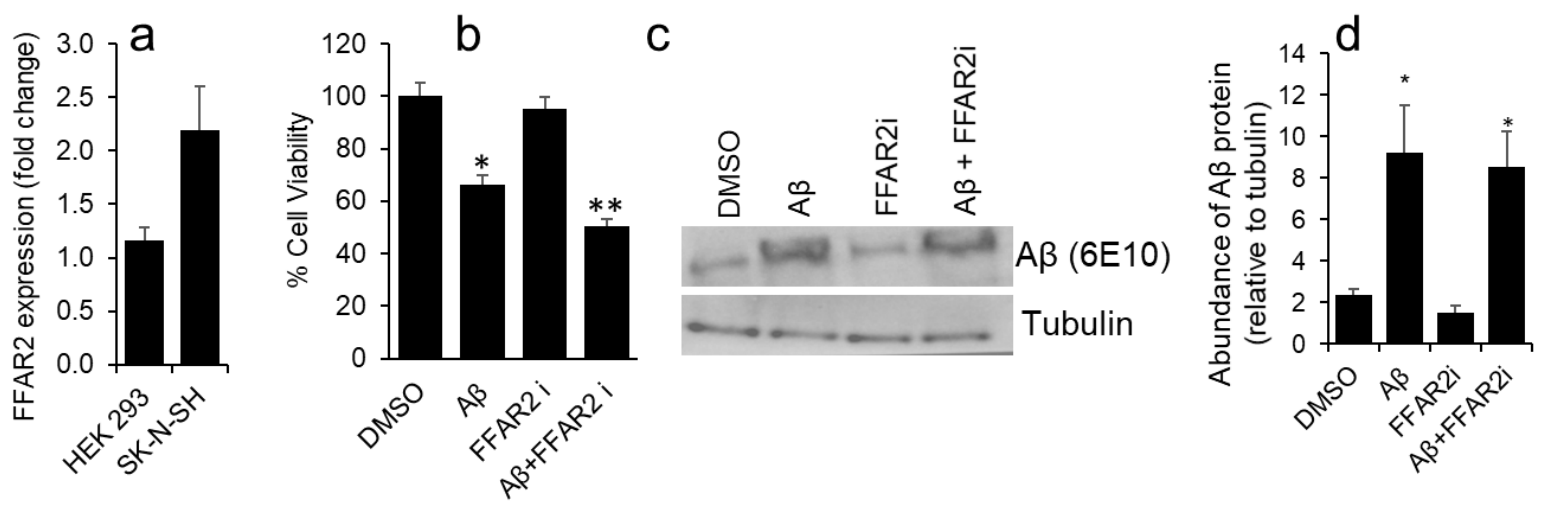

Figure 1. FFAR2 expressed in human neuronal cells and its inhibition increased $A \beta$-induced neurotoxicity. a) The mRNA expression of FFAR2 gene in SK-N-SH and HEK293 cells. b) SK-N-SH cells treated with FFAR2i (10 $\mu \mathrm{M}$ CATPB) for $4 \mathrm{~h}$ and A $325-35(25 \mu \mathrm{M})$ for $2 \mathrm{~h}$, then measured cell viability using MTT assay, show that A $\beta$-treatment induces cell death and inhibition of FFAR2 further reduced cell survival. DMSO used as vehicle control. c,d) Similarly, A $\beta$-treatment significantly increased A $\beta$-accumulation in both with and without FFAR2 inhibition. Data are the mean $(n=3$ replicates in 3 repeated experiments $) \pm$ SEM. $P$ values with ${ }^{*}<0.05$ and ${ }^{* *}<0.01$ are significantly different.

\subsection{In-silico screening discovered FFAR2 binding natural compounds}

The in-silico screening of $\sim 144,000$ natural compounds to see their binding affinity to active site of human FFAR2, we shortlisted top 15 compounds based on their binding energy and affinity (Supplementary Table S2). To determine the interactions of these selected natural compounds and predicting their agonist versus antagonists nature to FFAR2 and their interactions profiles were compared with known FFAR2 agonists like acetate and butyrate (Supplementary Tables S3a,b). The binding site residues Ser86, Tyr90, His140, Ile145, Val179, Arg180, Leu183, Tyr238, His242 and Arg255 
were retrieved from the previous publication [33]. By considering His242, Arg180 and Arg255 the key residues of human FFAR2 for agonist interactions of the ligands. It is observed that acetate forms H-bond with His242 in human FFAR2 and Arg65, Trp75, Tyr90, Gln148, Tyr238 in mouse FFAR2. For the butyrate, H-bond with His242 in human FFAR2 and Arg65, Trp75, Tyr90, Gln148, Tyr238 and Arg255 in mouse FFAR2 was observed. From the interaction analysis, it is observed that Fenchol (ZINC01081099) formed H-bond with the Ser86, Gln148, Glu166, Tyr238 and His242 of human FFAR2, Thr85 and Glu320 of mouse FFAR2. Residue His242 supports in organization of binding pocket for each agonist [34]. The lowest vina score of $-3 \mathrm{kcal} / \mathrm{mol},-3.2 \mathrm{kcal} / \mathrm{mol}$ and $-4.3 \mathrm{kcal} / \mathrm{mol},-3.8$ $\mathrm{kcal} / \mathrm{mol}$ was obtained for acetate and butyrate of human FFAR2 and mouse FFAR2 respectively. The lowest vina score of $-5.5 \mathrm{kcal} / \mathrm{mol}$ and $-5.3 \mathrm{kcal} / \mathrm{mol}$ for Fenchol was observed with house FFAR2 and mouse FFAR2 respectively (Figure 3a-d).

\subsection{Determining the FFAR2 agonist activity of selected compounds in neuronal cells}

To determine the FFAR2 agonist activity of selected 15 compounds, first, we analyzed toxicity profile and dose dependent effects of selected compounds, using MTT assay in SK-N-SH cells treated with various gradient concentrations $(10$ to $100 \mu \mathrm{M})$ and found that $10 \mu \mathrm{M}$ was the non-toxic and effective dose of most of the compounds (Supplementary Figure S2a). FFAR2 activation increases phosphorylation of tyrosine amino acid of ERK1/2 [35], therefore, while measuring phospho-ERK1/2 we found that Fenchol $>$ Octopamine $>$ Carvamenthenol $>$ Camphene $>$ Pentanone $>$ Synephrine $>$ Leucinamide $>$ Carene $>$ ๑-Terpineol $>$ Breneol $>$ L-Fenchone $>$ D-Fenchone $>$ Norpseudoephedrine $\mathrm{HCl}$ (Figure 2b) as showing the descending order of phospho-ERK1/2 expression on treatment to SK$\mathrm{N}-\mathrm{SH}$ cells. Moreover, levels of phosphor-ERK1/2 proteins were significantly higher in Fenchol treated cells compared to Acetate (a natural agonist of FFAR2), while these levels were lower in rest of the selected compound treated cells (Figure 2b; Supplementary Figure S2b). In addition, the

a Scheme of Screening and
validation FFAR2 agonists

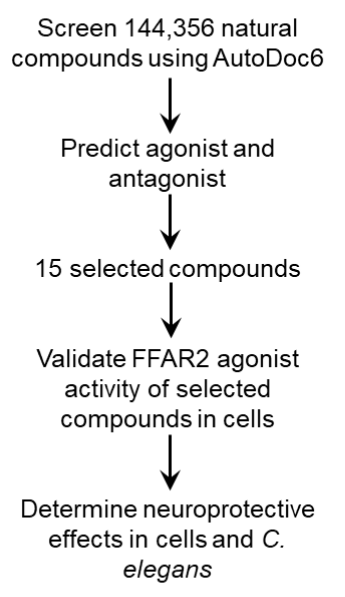

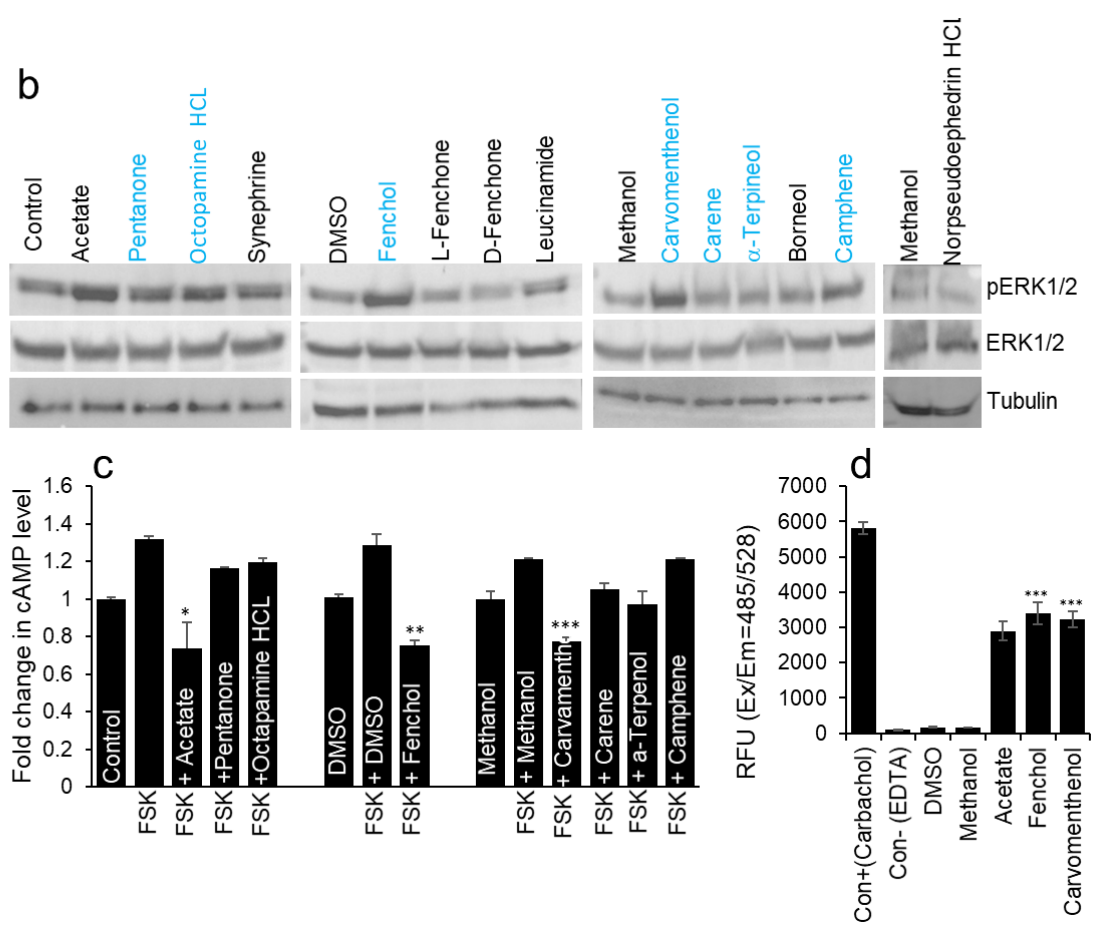

Figure 2. In-silico and in-vitro screening of FFAR2 agonist found Fenchol was most active to increase FFAR2 signaling via activating ERK1/2. a) Schematic of screening FFAR2 agonists and their validation for ameliorating AD-like phenotype. b) FFAR2 activation activity of selected compounds in terms of increasing phospho-ERK1/2 expression. c) Further selected compounds ( $n=7)$ compounds were showing reduction in Forskolin (FSK) induced cAMP in SK-N-SH cells. d) In addition, further selected $(\mathrm{n}=2)$ for increasing intracellular calcium- another indication of FFAR2 signaling activation. Data are shown as the mean \pm S.D. $\left(\mathrm{n}=3\right.$ experiments in triplicate). ${ }^{*} \mathrm{P}<0.05 ;{ }^{* *} \mathrm{P}<0.01 ;{ }^{* * *} \mathrm{P}<0.001,{ }^{* * *} \mathrm{P}<0.0001$ 
activation of FFAR2 results to stimulate Gi-signaling which in turn decreases intracellular cyclic adenosine mono-phosphate (cAMP) and increases calcium levels.[36] Interestingly, we found that Fenchol showed highest decrease in cAMP rise in SK-N-SH cells upon Forskolin-stimulation (an inducer of (AMP), and such decreases were shown in Carvamenthenol $<\alpha$-Terpineol $<$ Carene $<$ Pentanone < Octopamine < Camphene in an ascending order (Figure 2c). Further, Fenchol also showed highest increase in intracellular calcium levels in SK-N-SH cells (Figure 2d), suggesting that Fenchol functions like an agonist of human FFAR2 and stimulates Gi/o-signaling via cAMP-Ca2+ERK1/2 pathway.

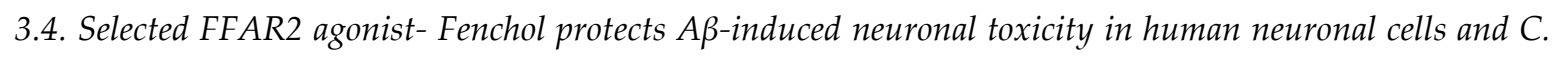
elegans

Further, structural analyses indicated that Fenchol and Acetate mimics in their binding to human and mouse FFAR2 (Figure 3a-d), further indicating that Fenchol binds to the active site to stimulate

a Interaction of acetate with human FFAR2

b Interaction of acetate with mouse FFAR2
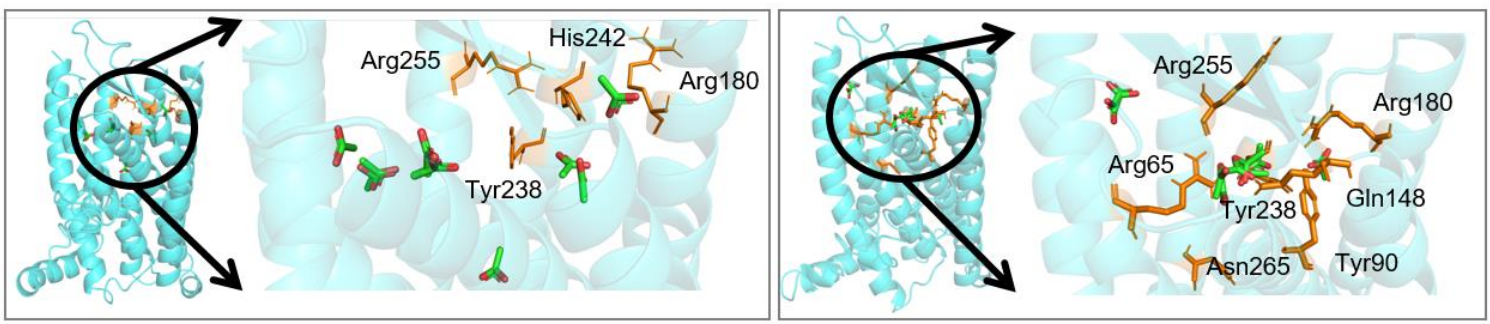

C Interaction of Fenchol with human FFAR2

d

Interaction of Fenchol with mouse FFAR2
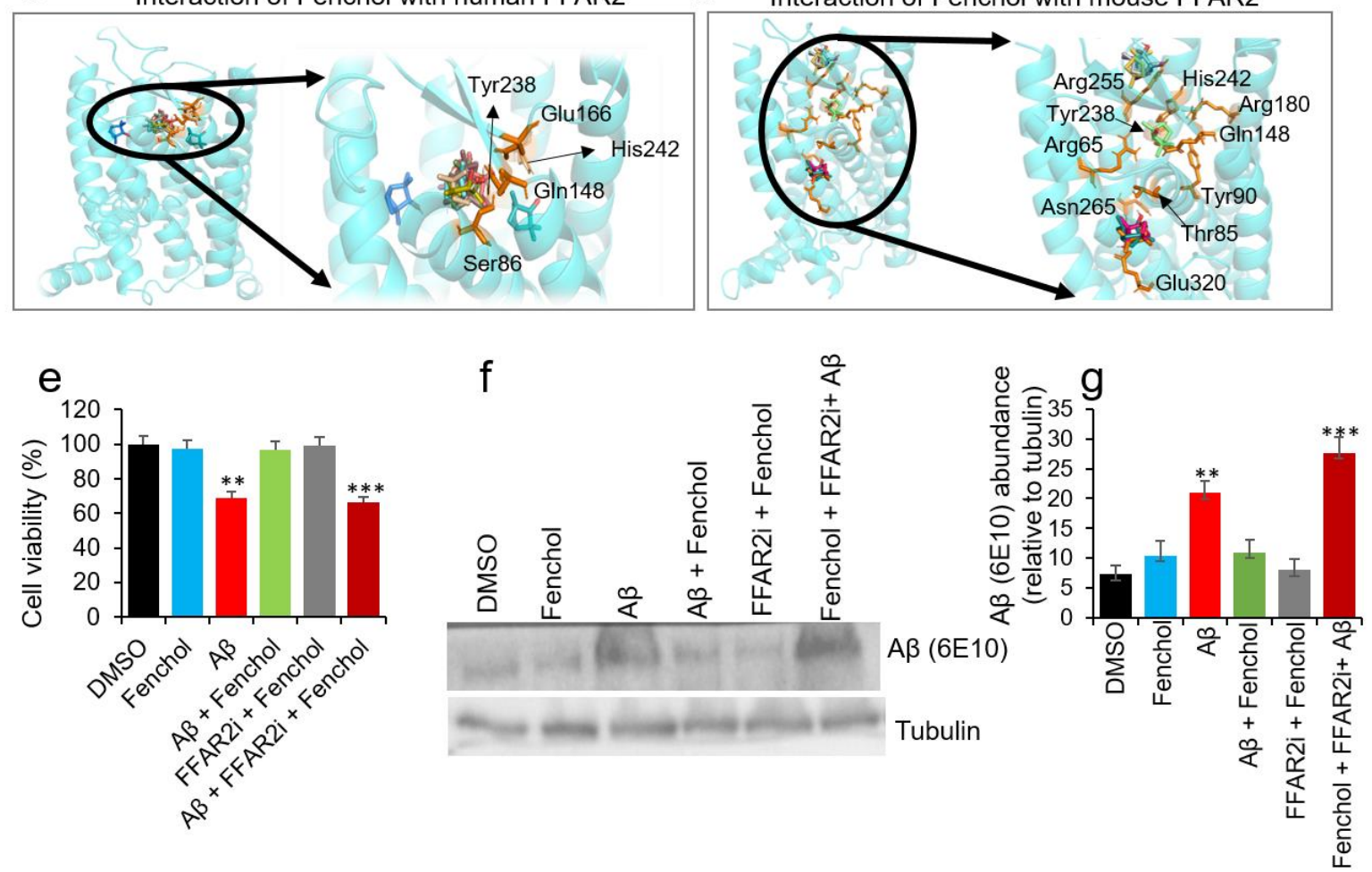

Figure 3. Fenchol binds to the FFAR2 active sites and activates FFAR2 signaling to exhibit neuroprotective effects. a-d) Interactions of Fenchol with human (c) and mouse (d) FFAR2 and its comparison with known FFAR2 agonist-Acetate $(a, b)$ in in-silico analyses. e) Fenchol treatment significantly reduced the A $\beta$ induced decline in cell viability (e) and A $\beta$-accumulation (f,g) in SK-N-SH cells on FFAR2 dependent manner. Data are mean \pm SEM. ( $\mathrm{n}=3$ intendent experiments in triplicate). ${ }^{*} \mathrm{P}<0.05 ;{ }^{* *} \mathrm{P}<0.01 ;{ }^{* * *} \mathrm{P}<0.001$, $* * * * \mathrm{P}<0.0001$ 
FFAR2 signaling. Further, we determined the effects of Fenchol treatment in the A $\beta$-induced neuronal cell death using SK-N-SH cells system and find that Fenchol treatment significantly reduced the A $\beta$-induced neuronal cell death in SK-N-SH cells compared to their controls during MTT assay (Figure 3e). Interestingly, the protective effects of Fenchol in A $\beta$-induced cell neurotoxicity was dependent on FFAR2 activity, as cells treated with FFAR2 inhibitor and Fenchol shows no significant protection in cell viability. Further, we show that Fenchol treatment significantly reduced A $\beta$ accumulation in SK-N-SH cells compared to their A $\beta$-only treated controls, again on FFAR2 dependent manner (Figure 3f,g), suggesting that Fenchol treatment reduced $A \beta$-accumulation in neuronal cells by activating FFAR2 signaling, thus prevents cell death.

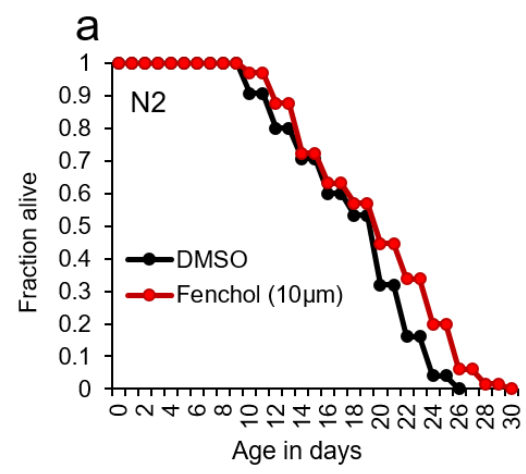

d
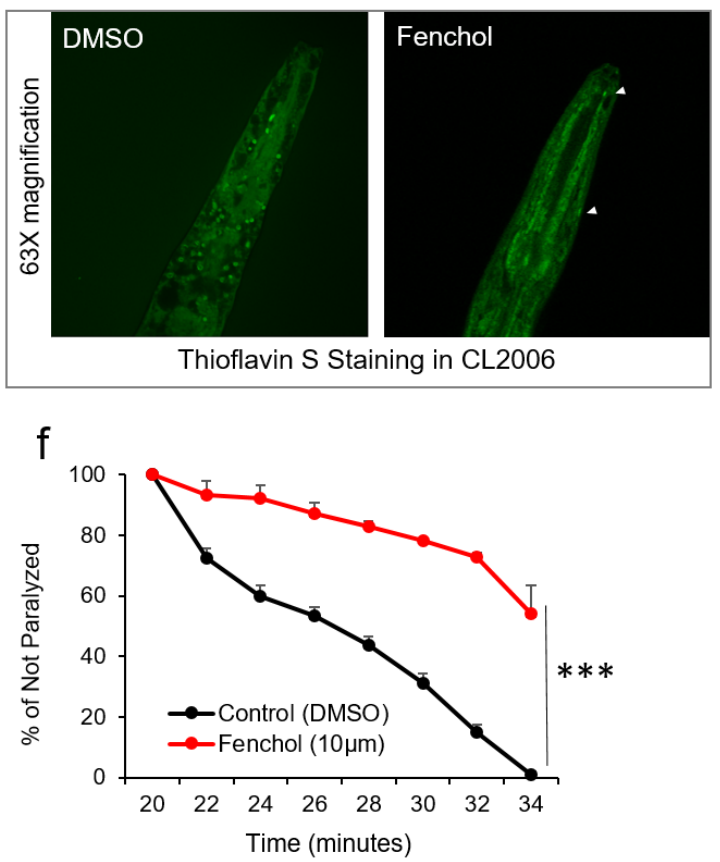
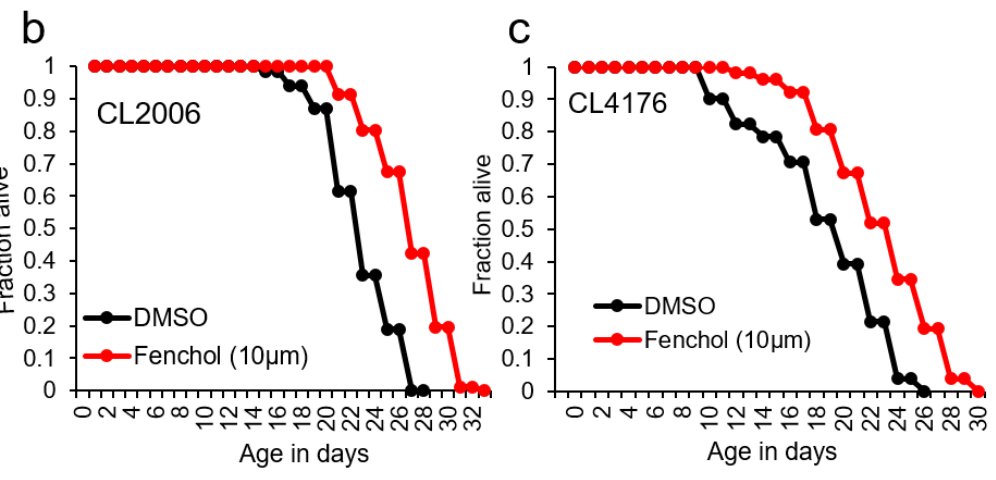

e
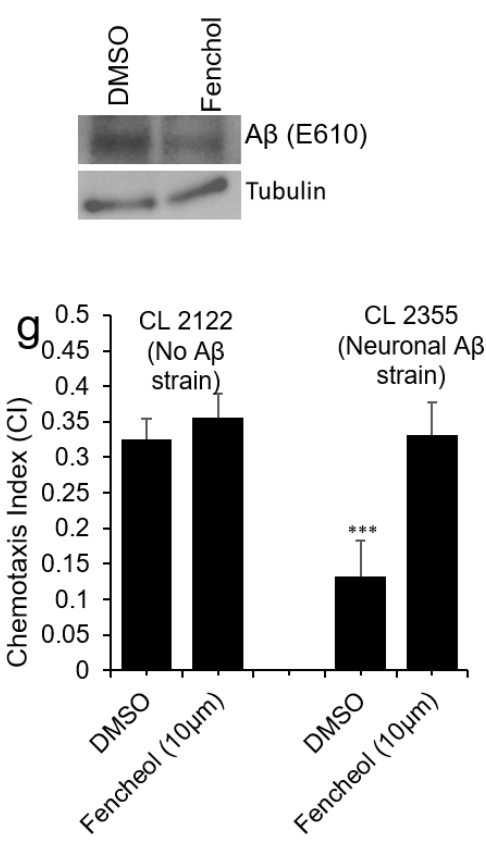

Figure 4. Fenchol increased prolongation of life-span in human $A \beta$-over-expressing, but not in wild-type $C$. elegans. a-c) Life-span assay of wild-type (a) and human A $\beta$-over-expressing (CL2006 and CL4176) (b,c) with and without Fenchol treatment. d,e) $A \beta$ accumulation shown by thioflavin $S$ staining (d) and western blot (e) in CL2006 worms treated with Fenchol in comparison to their DMSO treated controls. f) Fenchol treatment significantly protect temperature induced A $\beta$-mediated paralysis in CL4176 strains compared to their DMSO treated control group. g) In addition, Fenchol treatment also preserved temperature induced A $\beta$ overexpression mediated chemotaxis index (a markers of cognition/ memory) in CL2355 strain of C. elegans compared to their DMSO treated control, while Fenchol show normal effects in normal control CL2122 strain of C. elegans. Data are mean \pm SEM. ( $\mathrm{n}=3$ independent experiments in triplicate). 
To further demonstrate the physiological importance of Fenchol activity in reduced A $\beta$-induced neurotoxicity and AD like phenotypes, we used C. elegans as a model. First to demonstrate the impact of Fenchol treatment on life-span, the wild-type (N2) and transgenic worms expressing human A $\beta$ (CL2006 and CL4176) were used. Interestingly, we found that Fenchol treatment had no significant impact in the life-span of wild-type worms while significantly increased the survival/life-span in worms that over-expressing human A $\beta$ (CL2006 and CL4176) (Figure 4ab,c), suggesting that the Fenchol treatment have beneficial effects in prolongation of life by reducing $A \beta$ induced neurotoxicity in worms. Fenchol treatment significantly reduced $A \beta$-accumulation in human $A \beta-$ overexpressing worms (Figure $4 \mathrm{~d}, \mathrm{e}$ ), indicating that the beneficial effects of Fenchol are through reducing $A \beta$-accumulation. Fenchol treatment shows significant protection in neuronal health in over-expressing human $A \beta$ worms, as, Fenchol treated worms showed significantly reduced paralytic attacks in worms compared to their non-treated controls (Figure 4f). We also observed that Fenchol treatment significantly improved the memory behavior of over-expressing human $A \beta C$. elegans (CL2355) during chemotaxis assay compared to their non- $\mathrm{A} \beta$ expressing control worms (CL2122) (Figure 4g). Altogether, these results indicated that Fenchol improves overall survival, neuronal health and reduces neurotoxicity in cells and $C$. elegans by reducing $A \beta$-accumulation, but whether and how it reduces $A \beta$ accumulation still remain unknown.

\subsection{Fenchol increases proteasome activity to reduce A $\beta$-accumulation}

To determine how Fenchol can reduce the $\mathrm{A} \beta$ accumulation, we tested a hypothesis that Fenchol treatment increases $\mathrm{A} \beta$-clearance by increasing proteolytic activity. As, the primary protein
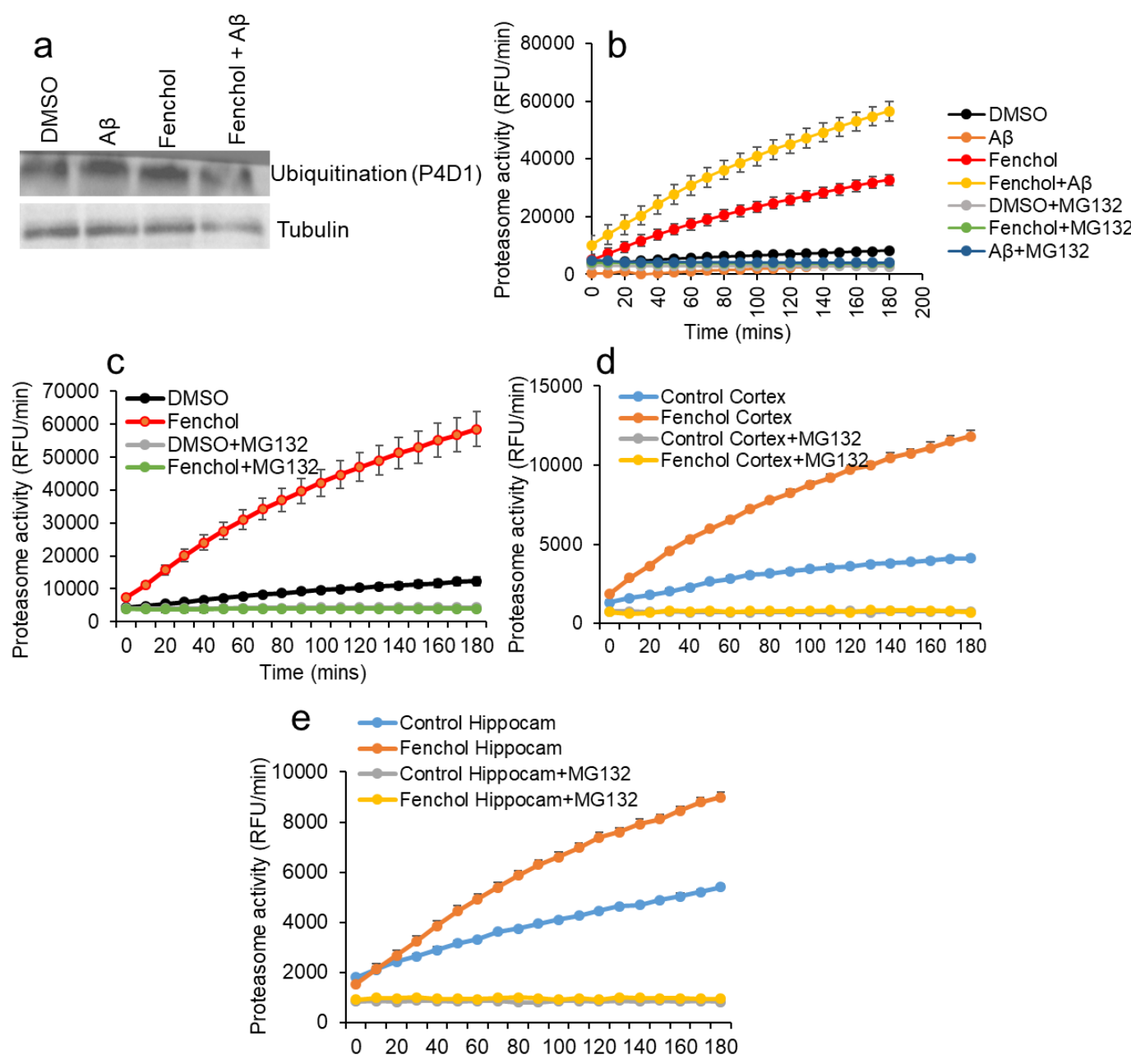

Figure 5. Fenchol treatment increase proteasome activity, without impacting protein ubiquitination. a) Total protein ubiquitination in Western blots with anti-P4D1 antibody was not impacted by Fenchol 
treatment. b,c) Proteasome activity was significantly increase in Fenchol treated SK-N-SH cells (b) and C. elegans (c). Proteasome activity was further higher in Fenchol treated SK-N-SH cells that were also treated with A $\beta$. Interestingly, Fenchol treatment also increased proteasome activity in APP/PS1 mice cortex (d) and hippocampus (e) regions. Data are three independent experiments done in triplicate and presented as Mean and SEM

degradation mechanisms are ubiquitination, we observed that Fenchol treatment did not impact that protein ubiquitinylation in the SK-N-SH cells (Figure 5a), suggesting that Fenchol effects were not mediated through degrading $A \beta$ due to increase ubiquitination. Further, we demonstrated that Fenchol treatment significantly increased the proteasomal activity in both SK-N-SH cells and C. elegans (Figure $5 b, c$ ). Interestingly, we also observed that these effects are not only restricted to cell lines and C. elegans, indeed, Fenchol treatment significantly increased the proteasomal activity in the cortex and hippocampus of brains of AD mouse model (APP/PS1). Altogether, these results indicate that the Fenchol activates proteasome activity in the neuronal cells to clear the $A \beta$-accumulation, thus reduces neurotoxicity in neuronal cells.

\subsection{Fenchol induces lysosomal activity and reduces cellular senescence in neuronal cells.}

Further, we show that Fenchol treatment significantly increased the lysosomal activity in the neuronal cells compared to their controls (Figure 6a). These results indicate that the Fenchol increases autophagy in the neuronal cells, thus might be protecting them from $A \beta$-induced neurotoxicity. We also observed that the $A \beta$-treated cells showed higher rate of senescence (indicated by $\beta$ galactosidase activity staining), while Fenchol treatment significantly reduced the number of senescence neuronal cells. These results further suggest that Fenchol's action involves in improving autophagy and reducing senescence in neurons, thus may protect neuronal death, and ameliorate AD like pathology in brain.
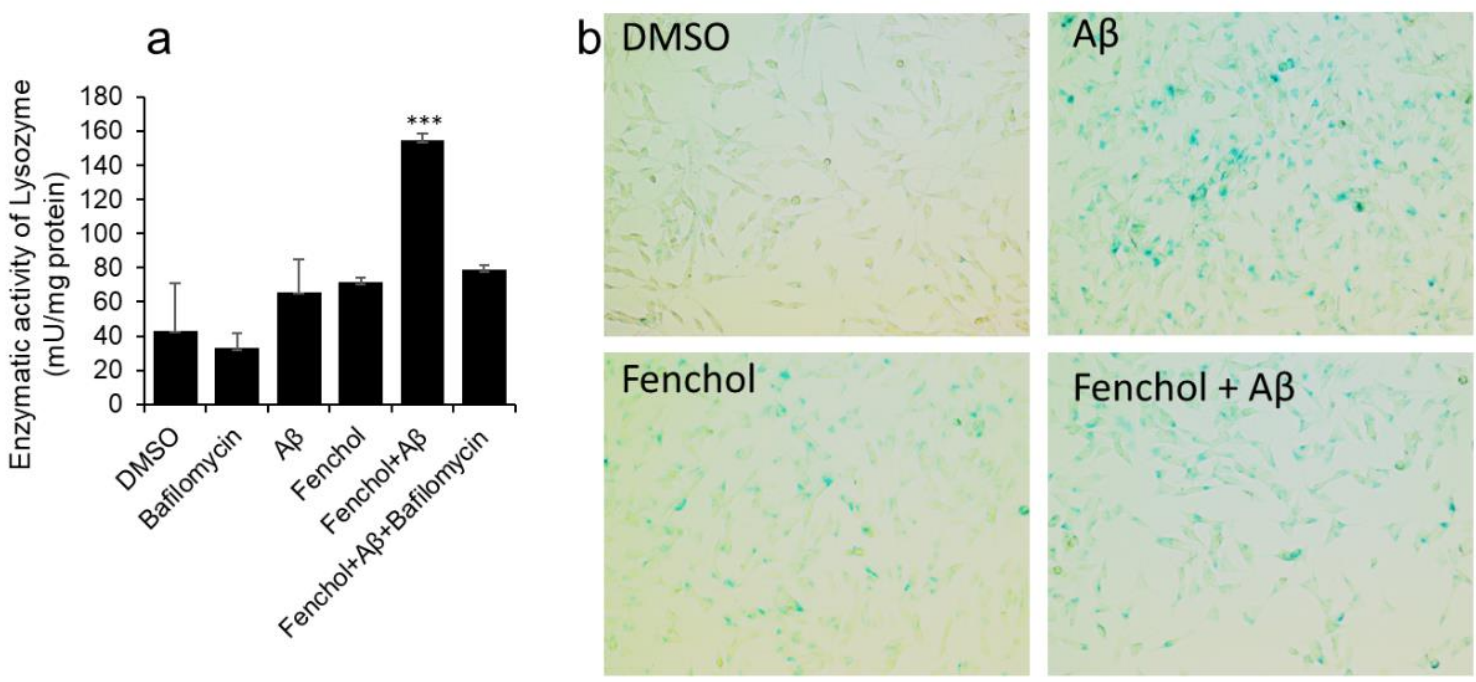

Figure 6. Fenchol treatment increases lysosomal activity (autophagy) (a) and reduces senescence (b) in SK$\mathrm{N}-\mathrm{SH}$ cells. Data are three independent experiments done in triplicate and presented as Mean and SEM.

\section{Discussion}

Emerging evidence indicate that the gut microbiota and its metabolites contributes in $\mathrm{AD}$ pathology, however mechanism(s) remain unknown. In addition, the precise targets by which microbiota sensing mechanisms can be manipulated to ameliorate AD pathology. Here, we 
demonstrated that microbiota derived metabolites like SCFAs signaling pathway called FFAR2 signaling contributes in A $\beta$-induced neurotoxicity and AD like phenotypes in human neuronal cells. Further, we also screened and evaluated natural compound- Fenchol that can stimulate FFAR2 signaling and ameliorate AD like phenotype in neuronal cells, C. elegans and mouse models. These results demonstrate FFAR2 as a potential target to modulate AD pathology and Fenchol can one of the natural compound to ameliorate $\mathrm{AD}$ by activating FFAR2 signaling.

FFAR2 is abundantly expressed not only in the intestinal tract cells, but it is also expressed in various other cell types including neurons, however, their function in neuronal cells are not well established. Here, we also demonstrated that the FFAR2 gene is prominently expressed in human neuronal (SK-N-SH) cells, further confirming its importance in neuronal cells and also establishes SK-N-SH cells as a model to study this signaling. FFAR2 is an inhibitory G (Gi/o)-coupled protein receptor signaling, and its activation initiated a signaling cascade of reducing intracellular cyclic AMP and increasing intracellular calcium and phosphorylation of ERK1/2 signaling. Thus, we used phospho-ERK1/2 activation (phosphorylation) as a primary screening for 15 selected natural compounds, following by reduction in cAMP and increase in calcium levels. Interestingly, Fenchol was found to be top-most FFAR2 signaling inducer in the human neuronal cells. Fenchol is commonly presents in edible plants like basil, grapes, mustard and some plant species like Aster; Alpinia Speciosa root oil [37,38]. It is also abundant in lavender oil, heart-wood and Eucalyptus; and commonly used in perfumes. Fenchol is known to exhibit antimicrobial, antioxidant and cell cytotoxic activities. However, its role in neuronal and neurological diseases remain largely unknown. We firsttime demonstrated its neuroprotective effects here in this study.

Accumulation of $A \beta$ is the hallmark of $A D$ pathology, and $A \beta$-induced neurotoxicity is one of the important mechanisms by which cognitive function and AD pathology prevails. Protecting neurons from $\mathrm{A} \beta$-induced cytotoxicity is an important target to prevent/treat AD pathology. FFAR2 inhibition increased the cytotoxic effects of $A \beta$ on neuronal cells, suggesting that activation of FFAR2 signaling may be neuroprotective. Interestingly, Fenchol treatment protected the $A \beta$-induced neuronal toxicity in SK-N-SH cells, and these effects were FFAR2 dependent manner, further suggesting that Fenchol protected neuronal cell death by activating FFAR2 signaling. C. elegans are emerging and commonly used model for several age-related conditions including AD-like phenotypes. Several C. elegans mutants have been developed to over-express human A $\beta$-proteins that make protein aggregates and human like $\mathrm{AD}$ or neurodegenerative pathology in worms. Here, we have used three $C$. elegans models such as wild-type (N2); CL2006- human A $\beta$ proteins overexpressing strain; CL4176- a temperature sensitive $A \beta$ expressing strain that grow and live normal at $16^{\circ} \mathrm{C}$ but develop paralysis due to over-expression of $A \beta$ and develop paralysis due to $A \beta$-induced neuronal death. In addition, we also used, CL2355- that overexpress human $A \beta$ while grown on room temperature at $22^{\circ} \mathrm{C}$, while grow normally at $16^{\circ} \mathrm{C}$, and compared the effects with a strain (CL2122) show normal chemotaxis (cognition/ memory). Interestingly, we found that Fenchol treatment significantly increased life-span in CL2006 and CL4176 strains, which was linked with significantly reduced $A \beta$-accumulation and $A \beta$-induced neurotoxicity mediated paralysis, respectively. We also show that $A \beta$-induced neurotoxicity mediated cognitive decline was also prevented in CL2355 strain of $C$. elegans. Altogether these results demonstrated that Fenchol treatment significantly reduced A $\beta-$ induced AD-like pathology in cells and C. elegans models, thus can be use as a neuroprotective agent to ameliorate $\mathrm{AD}$. 
Further, accumulation of $A \beta$ in brain and/or neurons are due to majorly reduced or defects in the protein degradation or clearance mechanisms, called proteolysis. Ubiquitination is common protein pathway by which older/non-functional proteins get ubiquitinylated, thus degraded in proteasomes. As, we show that Fenchol significantly reduced $A \beta$-accumulation in neuronal cell and $C$. elegans, but we found that this reduced $A \beta$ accumulation was not due to increased protein degradation through ubiquitination mediated $A \beta$ degradation. Thus, we analyzed the other mechanism(s) such as lysosomal degradation through autophagy, which contribute in efficient clearance of protein aggregates like $A \beta$ [39]. Interestingly, we found that Fenchol significantly increased the proteasomal/lysosomal activity in SK$\mathrm{N}-\mathrm{SH}$ cells and over-expressing human $\mathrm{A} \beta$ C. elegans. Although, as $C$. elegans have conserved human/ mammalian neuronal pathways [40,41], but to further confirm if Fenchol's mechanism of lysosomal activity is also conserved in mice; interesting, we found that the Fenchol also increases lysosomal activity in mouse brain. Higher $A \beta$-accumulation also induces cellular senescence in the neurons and

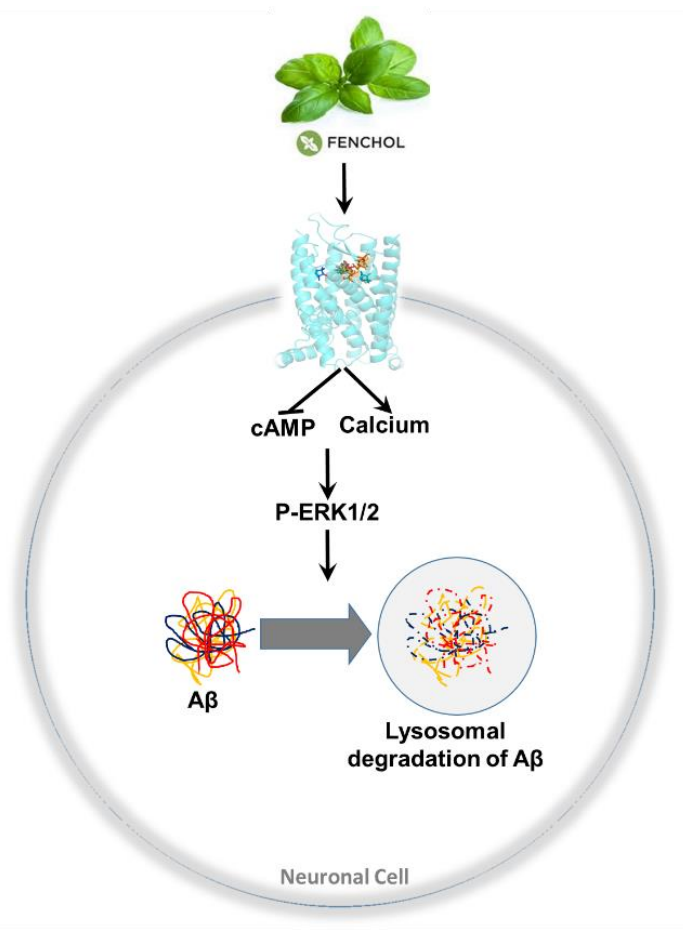
develop cognitive deficit/AD pathology [42-44]. We have observed similar phenomenon here in the SK-N-SH cells on being treated with $A \beta$, while the number of $A \beta$-induced senescent cells were significantly decreased after Fenchol treatment, suggesting that Fenchol reduced senescence in neuronal cells, thus have neuroprotective effects.

\section{Conclusions}

Our results demonstrated a microbiota sensor molecule- FFAR2 which mediates signaling of microbiota produced SCFAs plays an important role in the AD pathology by reducing A $\beta$-induced neuronal toxicity. Through in-silico large-scale drug screening of natural compounds library, invitro and in-vivo assays, we discovered that Fenchol- a common natural compound abundantly present in basil which functions like FFAR2 agonist, exhibits neuroprotective effects and ameliorate AD like pathology. Mechanistically, Fenchol increases $A \beta$-clearance by increasing proteasome and lysosomal activities, thus reduces $A \beta$-accumulation burden and protects $A \beta$-induced neuronal toxicity and senescence in neuronal cells. Our results indicated that the stimulation of FFAR2 and/or Fenchol can be used as a preventive and/or therapeutic strategy against AD.

Author Contributions: AR: performed experiments and analyzed data; PK SPM and SS: performed and helped bioinformatics analyses; SJ: contributed significantly in data interpretations, writing manuscripts and intellectual discussions; HY: conceived the idea, supervised the study, helped in data interpretations, developed and written manuscript, revised drafts of manuscripts. All authors reviewed and approved the final version of the manuscript.

Funding: We are thankful for the support provided by National Institutes of Health (NIH) grants R01AG018915; R56AG064075, R56AG069676; the Pepper Older Americans for Independence Center- P30AG21332, and the Department of Defense funding W81XWH-19-1-0236 (HY), and the NIH funded Wake Forest Alzheimer's Disease Research Center- P30AG049638 (SC) as well funds and services provided from the Center for Diabetes, Obesity and Metabolism, Wake Forest Baptist Medical Center, and the National Center for Advancing Translational Sciences (NCATS), the National Institutes of Health-funded Wake Forest Clinical and Translational Science Institute (WF CTSI) through Grant Award Number UL1TR001420. 
Acknowledgments: All the authors are grateful for all the fellow staff and laboratory members for their cooperation and help during the study.

Conflicts of Interest: : Dr. Yadav is Chief Scientific Officer and Cofounder of Postbiotics Inc, however, he has no conflict of interest for this work and his opinion in this article are fully independent and academic purposes. All other authors have nothing to disclose.

Appendix A: Please see supplementary tables and figures.

\section{References}

1. Singh, S.K.; Srivastav, S.; Yadav, A.K.; Srikrishna, S.; Perry, G. Overview of Alzheimer's Disease and Some Therapeutic Approaches Targeting Abeta by Using Several Synthetic and Herbal Compounds. Oxid Med Cell Longev 2016, 2016, 7361613, doi:10.1155/2016/7361613.

2. DeTure, M.A.; Dickson, D.W. The neuropathological diagnosis of Alzheimer's disease. Molecular Neurodegeneration 2019, 14, doi:10.1186/s13024-019-0333-5.

3. Yankner, B.A. Mechanisms of neuronal degeneration in Alzheimer's disease. Neuron 1996, 16, 921-932.

4. Selkoe, D.J. Alzheimer's disease: genes, proteins, and therapy. Physiological reviews 2001.

5. Liu, C.-C.; Zhao, N.; Yamaguchi, Y.; Cirrito, J.R.; Kanekiyo, T.; Holtzman, D.M.; Bu, G. Neuronal heparan sulfates promote amyloid pathology by modulating brain amyloid- $\beta$ clearance and aggregation in Alzheimer's disease. Science translational medicine 2016, 8, 332ra344-332ra344.

6. Settembre, C.; Fraldi, A.; Medina, D.L.; Ballabio, A. Signals from the lysosome: a control centre for cellular clearance and energy metabolism. Nature reviews Molecular cell biology 2013, 14, 283-296.

7. Leeman, D.S.; Hebestreit, K.; Ruetz, T.; Webb, A.E.; McKay, A.; Pollina, E.A.; Dulken, B.W.; Zhao, X.; Yeo, R.W.; Ho, T.T. Lysosome activation clears aggregates and enhances quiescent neural stem cell activation during aging. Science 2018, 359, 1277-1283.

8. He, N.; Jin, W.L.; Lok, K.H.; Wang, Y.; Yin, M.; Wang, Z.J. Amyloid- $31-42$ oligomer accelerates senescence in adult hippocampal neural stem/progenitor cells via formylpeptide receptor 2. Cell Death Dis 2013, 4, e924-e924, doi:10.1038/cddis.2013.437.

9. Nagpal, R.; Neth, B.J.; Wang, S.; Mishra, S.P.; Craft, S.; Yadav, H. Gut mycobiome and its interaction with diet, gut bacteria and alzheimer's disease markers in subjects with mild cognitive impairment: A pilot study. EBioMedicine 2020, 59, 102950, doi:10.1016/j.ebiom.2020.102950.

10. Nagpal, R.; Shively, C.A.; Register, T.C.; Craft, S.; Yadav, H. Gut microbiome-Mediterranean diet interactions in improving host health. F1000Res 2019, 8, 699, doi:10.12688/f1000research.18992.1.

11. Sun, J.; Xu, J.; Ling, Y.; Wang, F.; Gong, T.; Yang, C.; Ye, S.; Ye, K.; Wei, D.; Song, Z., et al. Fecal microbiota transplantation alleviated Alzheimer's disease-like pathogenesis in APP/PS1 transgenic mice. Transl Psychiat 2019, 9, doi:10.1038/s41398-019-0525-3.

12. Vogt, N.M.; Romano, K.A.; Darst, B.F.; Engelman, C.D.; Johnson, S.C.; Carlsson, C.M.; Asthana, S.; Blennow, K.; Zetterberg, H.; Bendlin, B.B., et al. The gut microbiota-derived metabolite trimethylamine N-oxide is elevated in Alzheimer's disease. Alzheimer's Research E Therapy 2018, 10, doi:10.1186/s13195018-0451-2.

13. Fujii, Y.; Nguyen, T.T.T.; Fujimura, Y.; Kameya, N.; Nakamura, S.; Arakawa, K.; Morita, H. Fecal metabolite of a gnotobiotic mouse transplanted with gut microbiota from a patient with Alzheimer's disease. Bioscience, Biotechnology, and Biochemistry 2019, 83, 2144-2152, doi:10.1080/09168451.2019.1644149. 
14. Zhuang, Z.; Gao, M.; Yang, R.; Liu, Z.; Cao, W.; Huang, T. Causal relationships between gut metabolites and Alzheimer's disease: a bidirectional Mendelian randomization study. Neurobiol Aging 2020, 10.1016/j.neurobiolaging.2020.10.022, doi:10.1016/j.neurobiolaging.2020.10.022.

15. Konjevod, M.; Nikolac Perkovic, M.; Sáiz, J.; Svob Strac, D.; Barbas, C.; Rojo, D. Metabolomics analysis of microbiota-gut-brain axis in neurodegenerative and psychiatric diseases. J Pharmaceut Biomed 2020, 10.1016/j.jpba.2020.113681, doi:10.1016/j.jpba.2020.113681.

16. Mishra, S.P.; Karunakar, P.; Taraphder, S.; Yadav, H. Free Fatty Acid Receptors 2 and 3 as Microbial Metabolite Sensors to Shape Host Health: Pharmacophysiological View. Biomedicines 2020, 8, doi:10.3390/biomedicines8060154.

17. Sun, J.; Yuan, B.; Wu, Y.; Gong, Y.; Guo, W.; Fu, S.; Luan, Y.; Wang, W. Sodium Butyrate Protects N2a Cells against A $\beta$ Toxicity In Vitro. Mediat Inflamm 2020, 2020, 1-9, doi:10.1155/2020/7605160.

18. Wenzel, T.J.; Gates, E.J.; Ranger, A.L.; Klegeris, A. Short-chain fatty acids (SCFAs) alone or in combination regulate select immune functions of microglia-like cells. Mol Cell Neurosci 2020, 105, doi:10.1016/j.mcn.2020.103493.

19. Nagpal, R.; Neth, B.J.; Wang, S.; Craft, S.; Yadav, H. Modified Mediterranean-ketogenic diet modulates gut microbiome and short-chain fatty acids in association with Alzheimer's disease markers in subjects with mild cognitive impairment. EBioMedicine 2019, 47, 529-542, doi:10.1016/j.ebiom.2019.08.032.

20. Wang, S.; Ahmadi, S.; Nagpal, R.; Jain, S.; Mishra, S.P.; Kavanagh, K.; Zhu, X.; Wang, Z.; McClain, D.A.; Kritchevsky, S.B., et al. Lipoteichoic acid from the cell wall of a heat killed Lactobacillus paracasei D35 ameliorates aging-related leaky gut, inflammation and improves physical and cognitive functions: from C. elegans to mice. Geroscience 2020, 42, 333-352, doi:10.1007/s11357-019-00137-4.

21. Ahmadi, S.; Wang, S.; Nagpal, R.; Wang, B.; Jain, S.; Razazan, A.; Mishra, S.P.; Zhu, X.; Wang, Z.; Kavanagh, K., et al. A human-origin probiotic cocktail ameliorates aging-related leaky gut and inflammation via modulating the microbiota/taurine/tight junction axis. JCI Insight 2020, 5, doi:10.1172/jci.insight.132055.

22. Ahmadi, S.; Razazan, A.; Nagpal, R.; Jain, S.; Wang, B.; Mishra, S.P.; Wang, S.; Justice, J.; Ding, J.; McClain, D.A., et al. Metformin reduces aging-related leaky gut and improves cognitive function by beneficially modulating gut microbiome/goblet cell/mucin axis. J Gerontol A Biol Sci Med Sci 2020, 10.1093/gerona/glaa056, doi:10.1093/gerona/glaa056.

23. Ahmadi, S.; Nagpal, R.; Wang, S.; Gagliano, J.; Kitzman, D.W.; Soleimanian-Zad, S.; Sheikh-Zeinoddin, M.; Read, R.; Yadav, H. Prebiotics from acorn and sago prevent high-fat-diet-induced insulin resistance via microbiome-gut-brain axis modulation. The Journal of Nutritional Biochemistry 2019, 67, 1-13, doi:10.1016/j.jnutbio.2019.01.011.

24. Nagpal, R.; Newman, T.M.; Wang, S.; Jain, S.; Lovato, J.F.; Yadav, H. Obesity-Linked Gut Microbiome Dysbiosis Associated with Derangements in Gut Permeability and Intestinal Cellular Homeostasis Independent of Diet. J Diabetes Res 2018, 2018, 3462092, doi:10.1155/2018/3462092.

25. Yang, J.; Yan, R.; Roy, A.; Xu, D.; Poisson, J.; Zhang, Y. The I-TASSER Suite: protein structure and function prediction. Nat Methods 2015, 12, 7-8, doi:10.1038/nmeth.3213.

26. Roy, A.; Kucukural, A.; Zhang, Y. I-TASSER: a unified platform for automated protein structure and function prediction. Nat Protoc 2010, 5, 725-738, doi:10.1038/nprot.2010.5.

27. Zhang, Y. I-TASSER server for protein 3D structure prediction. BMC Bioinformatics 2008, 9, 40, doi:10.1186/1471-2105-9-40. 
28. $\mathrm{Xu}, \mathrm{D}$.; Zhang, Y. Improving the physical realism and structural accuracy of protein models by a twostep atomic-level energy minimization. Biophys J 2011, 101, 2525-2534, doi:10.1016/j.bpj.2011.10.024.

29. Lovell, S.C.; Davis, I.W.; Arendall, W.B., 3rd; de Bakker, P.I.; Word, J.M.; Prisant, M.G.; Richardson, J.S.; Richardson, D.C. Structure validation by Calpha geometry: phi,psi and Cbeta deviation. Proteins 2003, 50, 437-450, doi:10.1002/prot.10286.

30. Trott, O.; Olson, A.J. AutoDock Vina: improving the speed and accuracy of docking with a new scoring function, efficient optimization, and multithreading. J Comput Chem 2010, 31, 455-461, doi:10.1002/jcc.21334.

31. Laskowski, R.A.; Swindells, M.B. LigPlot+: multiple ligand-protein interaction diagrams for drug discovery. J Chem Inf Model 2011, 51, 2778-2786, doi:10.1021/ci200227u.

32. Porta-de-la-Riva, M.; Fontrodona, L.; Villanueva, A.; Cerón, J. Basic Caenorhabditis elegans methods: synchronization and observation. JoVE (Journal of Visualized Experiments) 2012, e4019.

33. Schmidt, J.; Smith, N.J.; Christiansen, E.; Tikhonova, I.G.; Grundmann, M.; Hudson, B.D.; Ward, R.J.; Drewke, C.; Milligan, G.; Kostenis, E., et al. Selective orthosteric free fatty acid receptor 2 (FFA2) agonists: identification of the structural and chemical requirements for selective activation of FFA2 versus FFA3. J Biol Chem 2011, 286, 10628-10640, doi:10.1074/jbc.M110.210872.

34. Sergeev, E.; Hansen, A.H.; Pandey, S.K.; MacKenzie, A.E.; Hudson, B.D.; Ulven, T.; Milligan, G. Nonequivalence of key positively charged residues of the free fatty acid 2 receptor in the recognition and function of agonist versus antagonist ligands. Journal of Biological Chemistry 2016, 291, 303-317.

35. Liu, D.; Genetos, D.C.; Shao, Y.; Geist, D.J.; Li, J.; Ke, H.Z.; Turner, C.H.; Duncan, R.L. Activation of extracellular-signal regulated kinase (ERK1/2) by fluid shear is Ca2+-and ATP-dependent in MC3T3E1 osteoblasts. Bone 2008, 42, 644-652.

36. Al Mahri, S.; Al Ghamdi, A.; Akiel, M.; Al Aujan, M.; Mohammad, S.; Aziz, M.A. Free fatty acids receptors 2 and 3 control cell proliferation by regulating cellular glucose uptake. World Journal of Gastrointestinal Oncology 2020, 12, 514.

37. Singh, P.; Kalunke, R.M.; Giri, A.P. Towards comprehension of complex chemical evolution and diversification of terpene and phenylpropanoid pathways in Ocimum species. Rsc Adv 2015, 5, 106886106904, doi:10.1039/c5ra16637c.

38. Bleyer, W.A.; Sather, H.N.; Nickerson, H.J.; Coccia, P.F.; Finklestein, J.Z.; Miller, D.R.; Littman, P.S.; Lukens, J.N.; Siegel, S.E.; Hammond, G.D. Monthly pulses of vincristine and prednisone prevent bone marrow and testicular relapse in low-risk childhood acute lymphoblastic leukemia: a report of the CCG-161 study by the Childrens Cancer Study Group. J Clin Oncol 1991, 9, 1012-1021, doi:10.1200/JCO.1991.9.6.1012.

39. Lim, J.; Yue, Z. Neuronal aggregates: formation, clearance, and spreading. Dev Cell 2015, 32, 491-501, doi:10.1016/j.devcel.2015.02.002.

40. Chase, D.L.; Koelle, M.R. Biogenic amine neurotransmitters in C. elegans. WormBook 2007, 10.1895/wormbook.1.132.1, 1-15, doi:10.1895/wormbook.1.132.1.

41. Koelle, M.R. Neurotransmitter signaling through heterotrimeric G proteins: insights from studies in C. elegans. WormBook 2018, 10.1895/wormbook.1.75.2, 1-52, doi:10.1895/wormbook.1.75.2.

42. Wei, Z.; Chen, X.-C.; Song, Y.; Pan, X.-D.; Dai, X.-M.; Zhang, J.; Cui, X.-L.; Wu, X.-L.; Zhu, Y.-G. Amyloid $\beta$ Protein Aggravates Neuronal Senescence and Cognitive Deficits in 5XFAD Mouse Model of Alzheimer's Disease. Chinese Med J-Peking 2016, 129, 1835-1844, doi:10.4103/0366-6999.186646. 
43. Curtis, A. Targeting senescence within the Alzheimer's plaque. Science Translational Medicine 2019, 11, doi:10.1126/scitranslmed.aax4869.

44. Zhang, P.; Kishimoto, Y.; Grammatikakis, I.; Gottimukkala, K.; Cutler, R.G.; Zhang, S.; Abdelmohsen, K.; Bohr, V.A.; Sen, J.M.; Gorospe, M. Senolytic therapy alleviates A $\beta$-associated oligodendrocyte progenitor cell senescence and cognitive deficits in an Alzheimer's disease model. Nat Neurosci 2019, 22, 719-728. 